\title{
Efficient Feedback Methods for MIMO Channels Based on Parameterization
}

\author{
June Chul Roh, Member, IEEE, and Bhaskar D. Rao, Fellow, IEEE
}

\begin{abstract}
In this paper, we propose two efficient lowcomplexity quantization methods for multiple-input multipleoutput (MIMO) systems with finite-rate feedback based on proper parameterization of the information to be fed back followed by quantization in the new parameter domain. For a MIMO channel which has multiple orthonormal vectors as channel spatial information, we exploit the geometrical structure of orthonormality while quantizing the spatial information matrix. The parameterization is of two types: one is in terms of a set of unit-norm vectors with different lengths, and the other is in terms of a minimal number of scalar parameters. These parameters are shown to be independent for the i.i.d. flatfading Rayleigh channel, facilitating efficient quantization. In the first scheme, each of the unit-norm vectors is independently quantized with a finite number of bits using an optimal vector quantization (VQ) technique. Bit allocation is needed between the vectors, and the optimum bit allocation depends on the operating SNR of the system. In the second scheme, the scalar parameters are quantized. In slowly time-varying channels, the scalar parameters are also found to be smoothly changing over time, leading to the development of a simple quantization and feedback method using adaptive delta modulation. The results show that the proposed feedback scheme has a channel tracking feature and achieves a capacity very close to perfect feedback with a reasonable feedback rate.
\end{abstract}

Index Terms - Channel information feedback, channel state information, MIMO systems, multiple antennas, parameterization, quantization, transmit beamforming.

\section{INTRODUCTION}

$\mathbf{T}$ HE achievable performance of a multiple-antenna communication system relies heavily on the nature of channel state information (CSI) available at the transmitter and the receiver [1]. When the transmitter has perfect knowledge of the channel, a higher channel capacity and/or a better link performance can be achieved, and other benefits include lowercomplexity receiver and higher throughput in a multiuser environment. However, the perfect CSIT assumption could be unrealistic since in many practical systems the channel

Manuscript received March 30, 2005; revised January 11, 2006; accepted March 1, 2006. The associate editor coordinating the review of this paper and approving it for publication was V. Lau. This research was supported by UC Discovery Grant No. core02-10109 sponsored by Ericsson. The material in this paper was presented in part at the IEEE Wireless Communications and Networking Conference (WCNC) 2004, Atlanta, GA, March 2004, and at the IEEE International Symposium on Personal, Indoor and Mobile Radio Communications (PIMRC) 2004, Barcelona, Spain, September 2004.

J. C. Roh was with the Department of Electrical and Computer Engineering, University of California at San Diego, La Jolla, CA 92093-0407 USA. He is now with Texas Instruments, Inc., Sunnyvale, CA 94089 USA (e-mail: jroh@ti.com).

B. D. Rao is with the Department of Electrical and Computer Engineering, University of California at San Diego, La Jolla, CA 92093-0407 USA (e-mail: brao@ece.ucsd.edu).

Digital Object Identifier 10.1109/TWC.2007.05195 information is provided to the transmitter through a finite-rate feedback channel.

There are several related works dealing with how to feed back the multi-dimensional channel information. The problems of feeding back the transmit beamforming vector are studied in [2]-[7] (for multiple-input single-output (MISO) systems) and in [8] (for the principal eigen-mode feedback in MIMO systems). An important common feature in the proposed methods for beamforming vector feedback is that they are based on the inner-product between the actual and the quantized beamforming vector rather than the conventional mean squared error (MSE). It can be easily seen that the innerproduct is directly related to the received SNR and the channel capacity (see e.g. [5]).

Only recently, feedback of channel information in matrix forms for MIMO channels have begun to be addressed [9][15]. The problem in MIMO channels is much more complicated because of the multiple beamforming vectors. The CSI to be fed back is now of matrix form and in addition there is a need to provide information about power allocation over the multiple spatial channels. One promising approach is the joint quantization of the beamforming directions and power assignments by maximizing the average mutual information as studied in [11]. However, such a formulation leads to a difficult quantizer design problem. Using average mutual information as a measure directly does not lead to an iterative design algorithm with monotonic convergence property. In [13], a tractable measure of capacity loss due to finite feedback rate is derived under the assumption of equal power allocation, leading to an iterative quantizer design method with guaranteed monotonic convergence. One of practical issues with joint quantization (matrix quantization) using a full search vector quantization (VQ) is its high computational complexity (exponential in the number of feedback bits) in the encoding process. This can prevent real-time implementation and limit its use in practice. Motivated by complexity issues, lowcomplexity quantization issues have been a topic of extensive research in the source coding literature [16], [17]. A plethora of techniques are available that offer various options that tradeoff complexity to performance. Such considerations are also of interest in the MIMO feedback context, and in this paper we introduce two suboptimal feedback methods that offer good quantization performance with relatively low complexity. The first approach consists of applying a VQ technique developed for MISO channels in a sequential manner to quantize a MIMO channel. The second approach relates to the development of a simple channel feedback/tracking method for slowly time-varying MIMO channels. 
We use the following notations. $A^{\dagger}$ and $A^{T}$ indicate the conjugate transpose and the transpose of matrix $A$, respectively. $I_{n}$ is the $n \times n$ identity matrix and $0_{m, n}$ means the $m \times n$ zero matrix. $\operatorname{diag}\left(a_{1}, \ldots, a_{n}\right)$ is a square diagonal matrix with $a_{1}, \ldots, a_{n}$ along the diagonal. The inner-product between two vectors is defined as $\langle u, v\rangle=u^{\dagger} v$ and the 2-norm of vector $v$ is denoted by $\|v\|=\langle v, v\rangle^{1 / 2}$. $E[\cdot]$ represents the expectation operator, and $\tilde{\mathcal{N}}(\mu, \Sigma)$ is the proper complex Gaussian random vector with mean $\mu$ and covariance $\Sigma$. Uniform distribution over a set $S$ is denoted by $\mathcal{U}(S)$. An $m \times n(m \geq n)$ matrix $A$ with orthonormal columns, i.e., $A^{\dagger} A=I_{n}$, will be referred to as an orthonormal column matrix.

\section{SySTEM MODEL}

We consider a multiple antenna system with $t$ transmit and $r$ receive antennas. Assuming flat-fading, the MIMO channel is modeled by the channel matrix $H \in \mathbb{C}^{r \times t}$. That is, the channel input $x \in \mathbb{C}^{t}$ and the channel output $y \in \mathbb{C}^{r}$ have the following relationship:

$$
y=H x+\eta
$$

where $\eta$ is the additive white Gaussian noise vector distributed by $\tilde{\mathcal{N}}\left(0, I_{r}\right)$. The rank of $H$ is denoted by $m$. The singular value decomposition (SVD) of $H$ is given by (in economysize representation) $H=U_{H} \Sigma_{H} V_{H}^{\dagger}$, where $U_{H} \in \mathbb{C}^{r \times m}$ and $V_{H} \in \mathbb{C}^{t \times m}$ are orthonormal column matrices and $\Sigma_{H} \in$ $\mathbb{R}^{m \times m}$ contains the singular values $\sigma_{1} \geq \ldots \geq \sigma_{m}>0$ of $H$. The entries of $H$ are assumed to be i.i.d. $\tilde{\mathcal{N}}(0,1)$, complex circularly symmetric Gaussian with mean zero and variance 1 . The average transmit power is denoted by $P_{T}$, i.e., $E\left[x^{\dagger} x\right]=$ $P_{T}$.

We assume that perfect CSI is known to the receiver. For transmission of $n(1 \leq n \leq m)$ data streams, transmit beamforming along the $n$ principal eigenvectors of $H^{\dagger} H$ is the optimum choice in capacity sense [18]. Therefore, the first $n$ column vectors of $V_{H}$ are to be quantized and fed back to the transmitter as channel spatial information. We assume an error-free feedback channel with a finite rate of $B$ bits per channel update. For notational convenience, let us denote the $i$ th column vector of $V_{H}$ by $v_{i}$ and define the first $n$ column vectors of $V_{H}$ as $V$, i.e., $V=\left[v_{1}, \ldots, v_{n}\right]$.

The $t \times n$ quantized matrix $\hat{V}=\mathcal{Q}(V)$ is employed as the beamforming matrix at the transmitter. That is, an informationbearing symbol vector $s=\left[s_{1}, \ldots, s_{n}\right]^{T}$ is transmitted through $x=\hat{V} s$, resulting in the receive signal

$$
y=H \hat{V} s+\eta .
$$

Here we assume that $s \sim \tilde{\mathcal{N}}(0, \Phi)$ and $\Phi=P_{T}$. $\operatorname{diag}\left(\gamma_{1}, \ldots, \gamma_{n}\right)$ with $\gamma_{i} \geq 0$ and $\sum_{i} \gamma_{i}=1$. The vector $\gamma=$ $\left[\gamma_{1}, \ldots, \gamma_{n}\right]$ will be called power allocation information. With perfect knowledge of channel at the receiver, the optimum power allocation can be calculated at the receiver [19]. We will also consider quantizing and feeding back the power allocation information. The mutual information between $s$ and $y$ for a given channel $H$, when the transmitter uses the feedback channel information $(\hat{V}, \hat{\gamma})$ in transmission, is given by

$$
I_{\hat{V}, \hat{\gamma}}(H)=\log \operatorname{det}\left[I+\Sigma_{H}^{2} V_{H}^{\dagger} \hat{V} \Phi(\hat{\gamma}) V^{\dagger} V_{H}\right]
$$

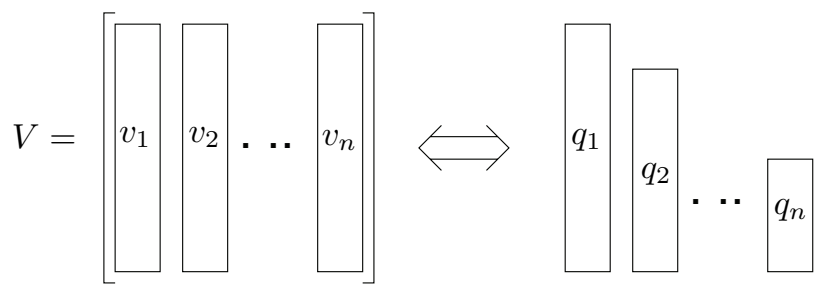

Fig. 1. Vector-form parameterization for spatial information. An orthonormal column matrix $V \in \mathbb{C}^{t \times n}$ can be uniquely represented by a set of unit-norm vectors, $q_{1} \in \mathcal{S}_{t}, q_{2} \in \mathcal{S}_{t-1}, \ldots, q_{n} \in \mathcal{S}_{t-n+1}$

where $\Phi(\hat{\gamma})$ is the power allocation associated with $\hat{\gamma}$, i.e., $\Phi(\hat{\gamma})=P_{T} \cdot \operatorname{diag}(\hat{\gamma})$.

Based on the fact that $\gamma$ depends essentially on the singular values of $H$ which are statistically independent of $V$, the vector $\gamma$ is quantized separately from $V$. A standard full search VQ is utilized for quantizing $\gamma$. The rest of the paper deals with quantizing $V$.

\section{Sequential Vector Quantization Technique FOR MIMO SYSTEMS}

In this section, we consider spatial channel information quantization for MIMO channels. As mentioned in Section II, the channel spatial information to be fed back is a $t \times n$ orthonormal column matrix $V=\left[v_{1}, \ldots, v_{n}\right](n \leq m \leq t)$. For ease of readability, we first summarize the key results that are the basis of the proposed quantization method and then describe the details of the method itself.

1) Parameterization: An orthonormal column matrix $V \in$ $\mathbb{C}^{t \times n}$ can be uniquely represented by a set of unit-norm vectors with different sizes, $q_{1} \in \mathcal{S}_{t}, q_{2} \in \mathcal{S}_{t-1}, \ldots$, $q_{n} \in \mathcal{S}_{t-n+1}$ (see Fig. 1), where $\mathcal{S}_{t}$ is the unit-norm sphere in $t$-dimensional space, i.e., $\mathcal{S}_{t}=\left\{u \in \mathbb{C}^{t}\right.$ : $\|u\|=1\}$.

2) Statistical Property: For a random channel $H$ with i.i.d. $\tilde{\mathcal{N}}(0,1)$ entries, each of the unit-norm vector is uniformly distributed over the corresponding unitnorm sphere, i.e., $q_{i} \sim \mathcal{U}\left(\mathcal{S}_{t-i+1}\right)$ for $i=1, \ldots, n$. Furthermore, the unit-norm vectors $q_{i}$ are statistically independent, motivating the sequential VQ scheme.

3) Sequential VQ (SVQ): For $i=1, \ldots, n$, the unit-norm vector $q_{i}$ is quantized sequentially using a codebook $\mathcal{C}_{i}$ that is designed for random unit-norm vector in $\mathcal{S}_{t-i+1}$ with the MSIP (mean squared inner-product) criterion [5].

The above claims and the method will be dealt with in Sections III-A, III-B and III-C, respectively. Before that, we briefly summarize the MSIP based VQ method for beamforming vector feedback, since it is used as a building block. Consider a MISO channel with $t$ transmit antennas. The channel matrix is a row vector: let us denote $H=h^{\dagger}\left(h \in \mathbb{C}^{t}\right)$. Its magnitude and direction are denoted as $\alpha=\|h\|$ and $v=h /\|h\|$, respectively, i.e., $h=\alpha v$. A quantized version of $v, \hat{v}=\mathcal{Q}(v)(\|\hat{v}\|=1)$, is employed as transmit beamforming vector. Then, the received signal $y$ is represented as

$$
y=\alpha\langle v, \hat{v}\rangle s+\eta
$$


where $s \in \mathbb{C}\left(E\left[|s|^{2}\right]=P_{T}\right)$ is the information-bearing transmit symbol. The received SNR is simply given by $\alpha^{2}|\langle v, \hat{v}\rangle|^{2} P_{T}$. Therefore, we can see that, for the channel with i.i.d. $\tilde{\mathcal{N}}(0,1)$ entries, maximizing $E[\mathrm{SNR}]$ is equivalent to maximizing $E|\langle v, \hat{v}\rangle|^{2}$, leading to the MSIP design criterion [4], [5]:

$$
\max _{\mathcal{Q}(\cdot)} E|\langle v, \mathcal{Q}(v)\rangle|^{2}
$$

One of virtues of the MSIP criterion is that it does lead to a closed-form VQ design algorithm; hence, an optimum codebook can be designed for any number of transmit antennas and any codebook size. A more general VQ design method especially for spatially correlated MISO channels can be found in [4], [5].

\section{A. Vector-Form Parameterization for Spatial Information}

We first present a lemma that will play an important role in the vector-form parameterization of the spatial information matrix $V$.

Lemma 1: Consider a $t \times t$ unitary matrix $G_{1}$ whose first column is $v_{1}$ (the first column of $V$ ) and the remaining columns are arbitrarily chosen to satisfy the orthonormality condition. Then the matrix $G_{1}^{\dagger} V$ has the following form:

$$
G_{1}^{\dagger} V=\left[\begin{array}{cc}
1 & 0 \\
0 & V^{(2)}
\end{array}\right]
$$

where $V^{(2)}$ is a $(t-1) \times(n-1)$ orthonormal column matrix and 0's are zero matrices with appropriate sizes.

Proof: Denote $G_{1}=\left[v_{1} \mid B\right]$, where $B$ is a $t \times(t-1)$ orthonormal column matrix and satisfies $v_{1}^{\dagger} B=0_{1, n-1}$; and also denote $V=\left[v_{1} \mid C\right]$. Then,

$$
G_{1}^{\dagger} V=\left[\begin{array}{c}
v_{1}^{\dagger} \\
B^{\dagger}
\end{array}\right]\left[\begin{array}{ll}
v_{1} & C
\end{array}\right]=\left[\begin{array}{cc}
v_{1}^{\dagger} v_{1} & v_{1}^{\dagger} C \\
B^{\dagger} v_{1} & B^{\dagger} C
\end{array}\right]=\left[\begin{array}{cc}
1 & 0 \\
0 & V^{(2)}
\end{array}\right] .
$$

The last equality is a consequence of the fact that $v_{1}$ is orthogonal to the remaining columns of $G_{1}$ and $V$, i.e., orthogonal to $B$ and $C$, by the orthonormality of the columns condition. Since $\left(G_{1}^{\dagger} V\right)$ is an orthonormal column matrix, $V^{(2)}$ must be a smaller orthonormal column matrix.

The unitary matrix $G_{1}$ can be defined deterministically as a function of $v_{1}$ in various ways, e.g., using Givens rotations and Householder reflections. We can recursively apply the above procedure to the smaller orthonormal column matrix $V^{(2)}$. That is, consider a $(t-1) \times(t-1)$ unitary matrix $\tilde{G}_{2}$ whose first column is the first column of $V^{(2)}$. Then, again by Lemma 1 ,

$$
\tilde{G}_{2}^{\dagger} V^{(2)}=\left[\begin{array}{cc}
1 & 0 \\
0 & V^{(3)}
\end{array}\right]
$$

where $V^{(3)}$ is a $(t-2) \times(n-2)$ orthonormal column matrix. Combining (6) and (5), we have

$$
G_{2}^{\dagger} G_{1}^{\dagger} V=\left[\begin{array}{cc}
I_{2} & 0 \\
0 & V^{(3)}
\end{array}\right], \text { where } G_{2}=\left[\begin{array}{cc}
1 & 0 \\
0 & \tilde{G}_{2}
\end{array}\right] .
$$

The above recursive procedure is continued until ones end up with $\tilde{I}=\left[I_{n}, 0_{n, t-n}\right]^{T}$.

Let us denote $V^{(1)}=V$ and the first column of $V^{(i)}$ by $q_{i}$ for $i=1, \ldots, n$. An important observation is that $G_{i}$ is a function only depending on $q_{i}$. Therefore, finally the original orthonormal column matrix $V$ is factored as

$$
V=G_{1}\left(q_{1}\right) G_{2}\left(q_{2}\right) \cdots G_{n}\left(q_{n}\right) \tilde{I},
$$

where the functional dependency of $G_{i}$ is explicitly indicated. With this factorization, we see that a $t \times n$ orthonormal column matrix $V$ can be uniquely represented by a set of unit-norm vectors, $q_{1} \in \mathcal{S}_{t}, q_{2} \in \mathcal{S}_{t-1}, \ldots, q_{n} \in \mathcal{S}_{t-n+1}$.

\section{B. Statistical Properties of the Parameter Vectors}

In this section, we discuss the statistical properties of the unit-norm vectors $\left\{q_{i}\right\}$. For this purpose, a few definitions are needed which are presented next. The set of all $t \times n$ $(t \geq n)$ complex matrices with orthonormal columns is called the complex Stiefel manifold, denoted by $\mathcal{V}_{n, t}=\{V(t \times n)$ : $\left.V^{\dagger} V=I_{n}\right\}$. When $n=1$, it is the unit-norm sphere, i.e., $\mathcal{V}_{1, t}=\mathcal{S}_{t}$. The following expression for the volume of the complex Stiefel manifold can be found in the literature (e.g., [20]):

$$
\operatorname{Vol}\left(\mathcal{V}_{n, t}\right)=\int_{\mathcal{V}_{n, t}} d V=\frac{2^{n} \pi^{n t}}{\tilde{\Gamma}_{n}(t)}
$$

where $\tilde{\Gamma}_{n}(a)$ is the complex multivariate gamma function given as $\tilde{\Gamma}_{n}(t)=\pi^{n(n-1) / 2} \prod_{k=1}^{n} \Gamma(t-k+1)$. For the unitnorm sphere,

$$
\operatorname{Vol}\left(\mathcal{S}_{t}\right)=\int_{\mathcal{S}_{t}} d v=\frac{2 \pi^{t}}{\Gamma(t)} .
$$

Let us consider the random matrix $V$ uniformly distributed over $\mathcal{V}_{n, t}$. The joint density function for $V$ is simply given by

$$
p(V)=1 / \operatorname{Vol}\left(\mathcal{V}_{n, t}\right), \quad \forall V \in \mathcal{V}_{n, t} .
$$

By the chain rule, the joint density function can be written in terms of the conditional density functions, i.e.,

$$
\begin{aligned}
p(V) & =p\left(v_{1}, v_{2}, \ldots, v_{n}\right) \\
& =p\left(v_{1}\right) \cdot p\left(v_{2} \mid v_{1}\right) \cdots p\left(v_{n} \mid v_{1}, \ldots, v_{n-1}\right) .
\end{aligned}
$$

Obviously, $v_{1}$ is uniformly distributed over $\mathcal{S}_{t}$, that is,

$$
p\left(v_{1}\right)=1 / \operatorname{Vol}\left(\mathcal{S}_{t}\right), \forall v_{1} \in \mathcal{S}_{t} .
$$

The conditional density functions are given as follows (see, e.g., [21]).

$$
p\left(v_{2} \mid v_{1}\right)=1 / \operatorname{Vol}\left(\mathcal{S}_{t-1}\right), \forall v_{2} \in \mathcal{S}_{t} \text { with } v_{2}^{\dagger} v_{1}=0 .
$$

Generally, for $i=1, \ldots, n$,

$$
\begin{aligned}
& p\left(v_{i} \mid v_{1}, \ldots, v_{i-1}\right)=1 / \operatorname{Vol}\left(\mathcal{S}_{t-i+1}\right), \\
& \forall v_{i} \in \mathcal{S}_{t} \text { with } v_{i}^{\dagger} v_{1}=0, \ldots, v_{i}^{\dagger} v_{i-1}=0
\end{aligned}
$$

Lemma 2: The volume expressions for the Stiefel manifold and the unit-norm spheres have the following relationship:

$$
\begin{aligned}
\operatorname{Vol}\left(\mathcal{V}_{n, t}\right) & =\operatorname{Vol}\left(\mathcal{S}_{t}\right) \cdot \operatorname{Vol}\left(\mathcal{S}_{t-1}\right) \cdots \operatorname{Vol}\left(\mathcal{S}_{t-n+1}\right) \\
& =\operatorname{Vol}\left(\mathcal{S}_{t}\right) \cdot \operatorname{Vol}\left(\mathcal{V}_{n-1, t-1}\right) .
\end{aligned}
$$

Proof: By substituting (11) and (14) into the chain rule expansion (12), we can easily arrive at the desired result. It can be also proved directly from the explicit volume expressions (9) and (10) using $\tilde{\Gamma}_{n}(t)=\left[\pi^{n-1} \Gamma(t)\right] \tilde{\Gamma}_{n-1}(t-1)$.

An important property of the random orthonormal column matrix whose distribution is given by (11) is the so-called 


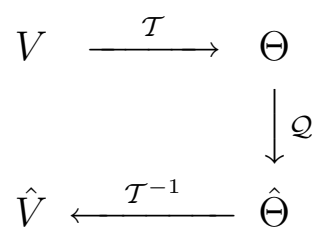

Fig. 2. Feedback strategy based on quantization in the parameter domain Parameterization: $\Theta=\mathcal{T}(V)$, Quantization: $\hat{\Theta}=\mathcal{Q}(\Theta)$, Reconstruction: $\hat{V}=\mathcal{T}^{-1}(\hat{\Theta})$.

unitary invariance property. That is, the probability density is unchanged when premultiplied by a deterministic unitary matrix $\Theta$, i.e.,

$$
p(V)=p\left(\Theta^{\dagger} V\right), \quad \forall V \in \mathcal{V}_{n, t} .
$$

The real-matrix counterpart is often called the Haar measure [22].

Utilizing the above lemmas, one can derive the following statistical properties of the vector-form parameters.

Theorem 1: Consider the first step of the parameterization in Section III-A for a $t \times n$ random matrix $V$ uniformly distributed over $\mathcal{V}_{n, t}$.

$$
G_{1}^{\dagger} V=\left[\begin{array}{cc}
1 & 0 \\
0 & V^{(2)}
\end{array}\right]
$$

Then, the matrix $V^{(2)}$ is uniformly distributed over $\mathcal{V}_{n-1, t-1}$ and is independent of $v_{1}$. That is, the conditional joint density function of $V^{(2)}$ for given $v_{1}$ is given by

$$
p\left(V^{(2)} \mid v_{1}\right)=1 / \operatorname{Vol}\left(\mathcal{V}_{n-1, t-1}\right), \forall V^{(2)} \in \mathcal{V}_{n-1, t-1} .
$$

Proof: When $V$ is premultiplied by $G_{1}^{\dagger}$, from the particular structure in the right hand side of (17), we can see that for given $v_{1}, p\left(G_{1}^{\dagger} V \mid v_{1}\right)=p\left(V^{(2)} \mid v_{1}\right)$. Using this fact, we can establish (18) as follows.

$$
\begin{aligned}
p\left(V^{(2)} \mid v_{1}\right) & =p\left(G_{1}^{\dagger} V \mid v_{1}\right) \\
& =p\left(V \mid v_{1}\right)=p\left(v_{2}, \ldots, v_{n} \mid v_{1}\right) \\
& =\frac{p(V)}{p\left(v_{1}\right)}=\frac{\operatorname{Vol}\left(\mathcal{S}_{t}\right)}{\operatorname{Vol}\left(\mathcal{V}_{n, t}\right)} \\
& =1 / \operatorname{Vol}\left(\mathcal{V}_{n-1, t-1}\right), \quad \forall V^{(2)} \in \mathcal{V}_{n-1, t-1}
\end{aligned}
$$

where (19) is a result of the unitary invariant property, (20) comes from the relationship $p(V)=p\left(v_{1}\right) \cdot p\left(v_{2}, \ldots, v_{n} \mid v_{1}\right)$ and the uniform density expressions in (11) and (13), and finally (21) from the relationship between the volume expressions given in Lemma 2.

By applying the above theorem recursively as in Section IIIA, we can arrive at the desired result stated as item 2) earlier in this section.

\section{The Sequential Vector Quantization (SVQ) Method}

The general feedback strategy ${ }^{1}$ is depicted in Fig. 2 and summarized below.

1. From the spatial information $V$, extract a set of parameters $\Theta$ that has a one-to-one mapping to $V$ (Parameterization): $\Theta=\mathcal{T}(V)$.

\footnotetext{
${ }^{1}$ The same framework is used in developing the feedback method that will be introduced in Section IV.
}

TABLE I

The Sequential Vector Quantization Method for MiMO

CHANNELS

\begin{tabular}{l|c|c}
\hline Parameterize at receiver & Quantize & Reconstruct at transmitter \\
\hline$v_{1}=q_{1}$ & $\hat{q}_{1}=\mathcal{Q}_{1}\left(q_{1}\right)$ & $\hat{v}_{1}=\hat{q}_{1}$ \\
$G_{1}^{\dagger} v_{2}=\left[\begin{array}{c}0 \\
q_{2}\end{array}\right]$ & $\hat{q}_{2}=\mathcal{Q}_{2}\left(q_{2}\right)$ & $\hat{v}_{2}=\hat{G}_{1}\left[\begin{array}{c}0 \\
\hat{q}_{2}\end{array}\right]$ \\
$\vdots$ & $\vdots$ & $\vdots$ \\
$G_{n-1}^{\dagger} \cdots G_{1}^{\dagger} v_{n}=\left[\begin{array}{c}0 \\
q_{n}\end{array}\right]$ & $\hat{q}_{n}=\mathcal{Q}_{n}\left(q_{n}\right)$ & $\hat{v}_{n}=\hat{G}_{1} \cdots \hat{G}_{n-1}\left[\begin{array}{c}0 \\
\hat{q}_{n}\end{array}\right]$ \\
\hline
\end{tabular}

2. Quantize the parameters $\Theta$ and feed back the quantized version $\hat{\Theta}$ to the transmitter (Quantization): $\hat{\Theta}=\mathcal{Q}(\Theta)$.

3. Reconstruct the spatial information $\hat{V}$ from $\hat{\Theta}$ (Reconstruction): $\hat{V}=\mathcal{T}^{-1}(\hat{\Theta})$.

The parameter set $\Theta$ is $\left\{q_{1}, \ldots, q_{n}\right\}$ in this section. The basic idea of the proposed SVQ method is to quantize the unitnorm vectors $\left\{q_{i}\right\}$ independently using a corresponding set of codebooks $\left\{\mathcal{C}_{i}\right\}$. The codebook $\mathcal{C}_{i}$ is designed for random unitnorm vector in $\mathcal{S}_{t-i+1}$ as described at the beginning of the section. The independence of the unit-norm vectors (Theorem 1) indicates that the overall loss using the SVQ method is minimal compared to joint quantization of $\left\{q_{i}\right\}$.

Table I summarizes the overall procedure. Equivalent vector-wise notations are used since we can see the relations between $\left\{v_{i}\right\}$ and $\left\{q_{i}\right\}$ more clearly. Generally, the parameterization, the quantization, and the reconstruction procedures are described, respectively, as follows: for $i=1, \ldots, n$,

$$
\begin{gathered}
G_{i-1}^{\dagger} \cdots G_{1}^{\dagger} v_{i}=\left[\begin{array}{c}
0 \\
q_{i}
\end{array}\right], \\
\hat{q}_{i}=\mathcal{Q}_{i}\left(q_{i}\right), \\
\hat{v}_{i}=\hat{G}_{1} \cdots \hat{G}_{i-1}\left[\begin{array}{c}
0 \\
\hat{q}_{i}
\end{array}\right],
\end{gathered}
$$

where $\mathcal{Q}_{i}(\cdot)$ represents quantization over codebook $\mathcal{C}_{i}$ (designed for random vector in $\mathcal{S}_{t-i+1}$ ), and $\hat{G}_{i}$ is the unitary matrix generated from $\hat{q}_{i}$ in the same manner as that used in generating $G_{i}$ from $q_{i}$. The quantized version of $V$ can be also written as

$$
\hat{V}=\hat{G}_{1}\left(\hat{q}_{1}\right) \hat{G}_{2}\left(\hat{q}_{2}\right) \cdots \hat{G}_{n}\left(\hat{q}_{n}\right) \tilde{I} .
$$

Note that the reconstructed matrix $\hat{V}$ has the same geometrical structure (orthonormal columns) as the original $V$.

In selecting the unit-norm vector $\hat{q}_{i}$ among the candidate vectors in a given codebook $\mathcal{C}_{i}$, we consider the following encoding schemes with different levels of complexity.

Encoding Scheme A: Encode sequentially $i=1, \ldots, n$ as

$$
\hat{q}_{i}=\arg \max _{\hat{q} \in \mathcal{C}_{i}}\left|\left\langle q_{i}, \hat{q}\right\rangle\right| .
$$

This simple scheme is expected to work well when the number of feedback bits $B$ is large. However, when $B$ is small we confront some error propagation problems. This can be seen in the reconstruction procedure. The $i$ th reconstructed vector $\hat{v}_{i}$ is dependent not only on $\hat{q}_{i}$, but also on the previously decoded vectors $\hat{q}_{1}, \ldots, \hat{q}_{i-1}$. Therefore, quantization errors in $\hat{q}_{1}, \ldots, \hat{q}_{i-1}$ accumulate and affect the error associated with the current vector $\hat{v}_{i}$. To alleviate this effect, we consider a 
new encoding scheme whose criterion is maximizing $\left|\left\langle v_{i}, \hat{v}_{i}\right\rangle\right|$ after the vector $\hat{v}_{i}$ is reconstructed using $\hat{q}_{1}, \ldots, \hat{q}_{i}$.

Encoding Scheme B: Encode sequentially $i=1, \ldots, n$ as

$$
\hat{q}_{i}=\arg \max _{\hat{q} \in \mathcal{C}_{i}}\left|\left\langle v_{i}, \hat{v}_{i}\right\rangle\right|
$$

where $\hat{v}_{i}=\hat{G}_{1} \cdots \hat{G}_{i-1}\left[\begin{array}{ll}0 & \hat{q}\end{array}\right]^{T}$. It is easy to see that (27) is equivalent to the following:

$$
\hat{q}_{i}=\arg \max _{\hat{q} \in \mathcal{C}_{i}}\left|\left\langle\tilde{q}_{i}, \hat{q}\right\rangle\right|
$$

where $\tilde{q}_{i}$ is related with $v_{i}$ as $\hat{G}_{i-1}^{\dagger} \cdots \hat{G}_{1}^{\dagger} v_{i}=\left[\begin{array}{ll}a^{T} & \tilde{q}_{i}^{T}\end{array}\right]^{T}$, for some $a \in \mathbb{C}^{i-1}$.

The third encoding scheme, described next, results in better performance but with higher computational complexity. Consider the composite codebook $\mathcal{C}$ that contains all the possible beamforming vectors generated from $\mathcal{C}_{1}, \mathcal{C}_{2}, \ldots, \mathcal{C}_{n}$.

$$
\mathcal{C}=\left\{V=\mathcal{T}^{-1}\left(\hat{q}_{1}, \ldots, \hat{q}_{n}\right): \hat{q}_{1} \in \mathcal{C}_{1}, \ldots, \hat{q}_{n} \in \mathcal{C}_{n}\right\}
$$

where $\mathcal{T}: \mathcal{V}_{n, t} \rightarrow \mathcal{S}_{t} \times \ldots \times \mathcal{S}_{t-n+1}$ represents the parameterization for spatial information matrix and $\mathcal{T}^{-1}$ denotes the reconstruction mapping. The cardinality of the composite codebook $|\mathcal{C}|=\left|\mathcal{C}_{1}\right| \times\left|\mathcal{C}_{2}\right| \times \cdots \times\left|\mathcal{C}_{n}\right|$.

Encoding Scheme $\mathbf{C}$ : Encode to maximize the mutual information as

$$
\hat{V}=\arg \max _{\hat{W} \in \mathcal{C}}\left\{\max _{\gamma} \log \operatorname{det}\left[I+H \hat{W} \Phi(\gamma) \hat{W}^{\dagger} H\right]\right\} .
$$

The computational complexity of an encoding scheme is mainly determined by the search complexity (the number of code point comparisons to find the best one in the codebook). For encoding scheme $\mathrm{A}$ and $\mathrm{B}$, the search complexity is proportional to $\sum_{i=1}^{n}\left|\mathcal{C}_{i}\right|$, compared to $\prod_{i=1}^{n}\left|\mathcal{C}_{i}\right|=2^{B}$ for encoding scheme $\mathrm{C}$. It is easy to see that the former is always less than the later. For example, when $\left|\mathcal{C}_{i}\right|=N_{0}$ for all $i$, the search complexity of encoding scheme $\mathrm{A}$ and $\mathrm{B}$ is linearly with respect to $n$ as $n N_{0}$, while for encoding scheme $\mathrm{C}$ it grows exponentially as $N_{0}^{n}$. As will be shown later, encoding scheme $\mathrm{C}$ requires the least number of feedback bits for a given target performance at the a cost of increased computational complexity.

\section{Design Examples and Comparisons With Other Methods}

The multiple spatial channels in MIMO channels have different contributions to the channel capacity since each spatial channel has a different channel gain. This should be also taken into account while designing a feedback systems, leading to the problem of bit allocation. We will discuss this problem with a simulation study. We considered a $(t=4, r=$ 2) MIMO channel with i.i.d. $\tilde{\mathcal{N}}(0,1)$ entries, which has a $4 \times 2$ orthonormal column matrix for feedback. Using the parameterization method, we extract two unit-norm vectors, $q_{1} \in \mathbb{C}^{4}$ and $q_{2} \in \mathbb{C}^{3}$. Then, we quantize $q_{1}$ and $q_{2}$ using codebooks $\mathcal{C}_{1}$ and $\mathcal{C}_{2}$, respectively. The two codebooks could have different codebook sizes, and the total number of quantization/feedback bits is assumed to be fixed at $B=8$. There are many possible ways of allocating $B$ bits in quantizing the unit-norm vectors $\left(q_{1} \in \mathcal{S}_{4}\right.$ and $q_{2} \in \mathcal{S}_{3}$ ) and power allocation $\gamma$. We considered the following cases in the simulations:

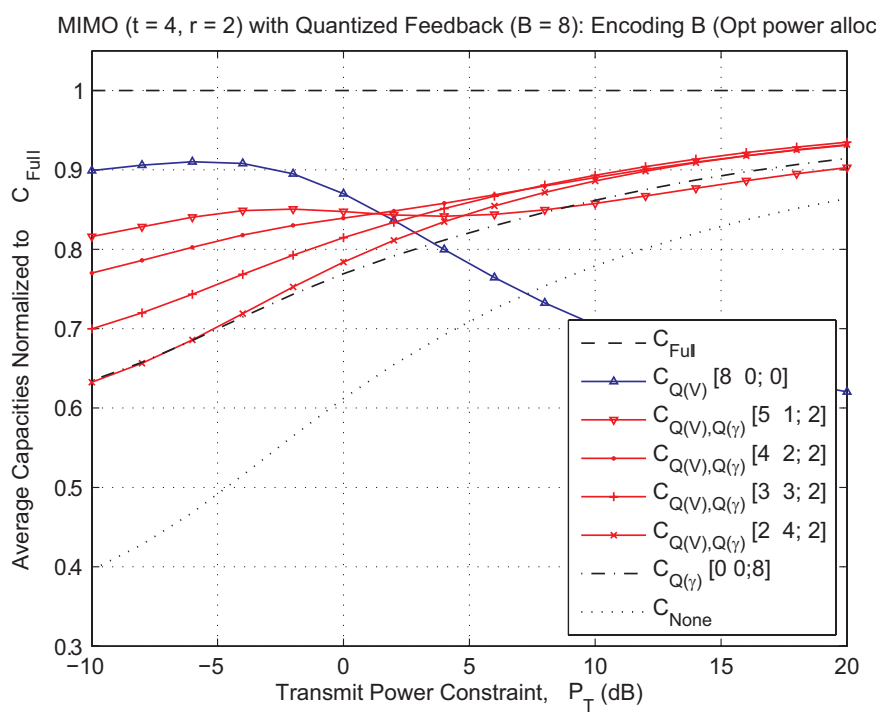

Fig. 3. Normalized average capacities of MIMO channel with quantized feedback and different bit allocations: encoding scheme $\mathrm{B}(t=4, r=2$ and $B=8)$.

$\left[B_{1} B_{2} ; B_{\gamma}\right]=[80 ; 0],[51 ; 2],[42 ; 2],[33 ; 2],[24 ; 2],[00 ; 8]$, where $B_{i}$ is the number of bits allocated for $q_{i}$ and $B_{\gamma}$ for $\gamma$ with $B_{1}+B_{2}+B_{\gamma}=B$. For example, the first case $[80 ; 0]$ means that all the bits are used to quantize only $q_{1}$ and no information about $q_{2}$ is fed back (i.e., quantized maximal ratio transmission scheme). The MSE VQ design method is used to design the codebooks for power allocation wherever appropriate.

We first present results with the encoding scheme B which provides good performance with reasonable complexity. Fig. 3 shows the average capacities with different bit allocations. For ease of comparison, all the capacities have been normalized to the capacity with the complete CSIT $\left(C_{\text {Full }}\right)$, and therefore a one on the plots indicates what is possible with perfect feedback. We can see that the optimum bit allocation is dependent on the SNR at which the system is operating. In the low SNR region, the optimum strategy is to quantize only the first unit-norm vector $q_{1}$, i.e., $\left[B_{1} B_{2} ; B_{\gamma}\right]=[80 ; 0]$. As the SNR increases, it changes to $[42 ; 2]$; and in the high power region, the optimum bit allocation is $[33 ; 2]$.

Another alternate bit allocation strategy is to use all available feedback bits to quantize only spatial information, and employ equal power allocation in transmission. From simulation results shown in Fig. 4, we can see that it performs better in the high SNR region. This is because when the SNR is high, equal power allocation is close to optimum in most cases, therefore resulting in no wasted bits for power allocation.

In the literature, mainly two other codebook construction methods have been studied: i) an approach based on Grassmannian subspace packing [12]; and ii) matrix quantization (MQ) based codebook construction [11], [13], [14]. In [12], similarity between quantized beamforming and unitary spacetime constellation design is first addressed, and the discrete Fourier transform (DFT) matrix based design algorithm proposed in [23] is adopted in the beamforming codebook design. In MQ-based approach, the codebook is designed via a Lloydtype algorithm, which is similar to vector quantization (VQ) 


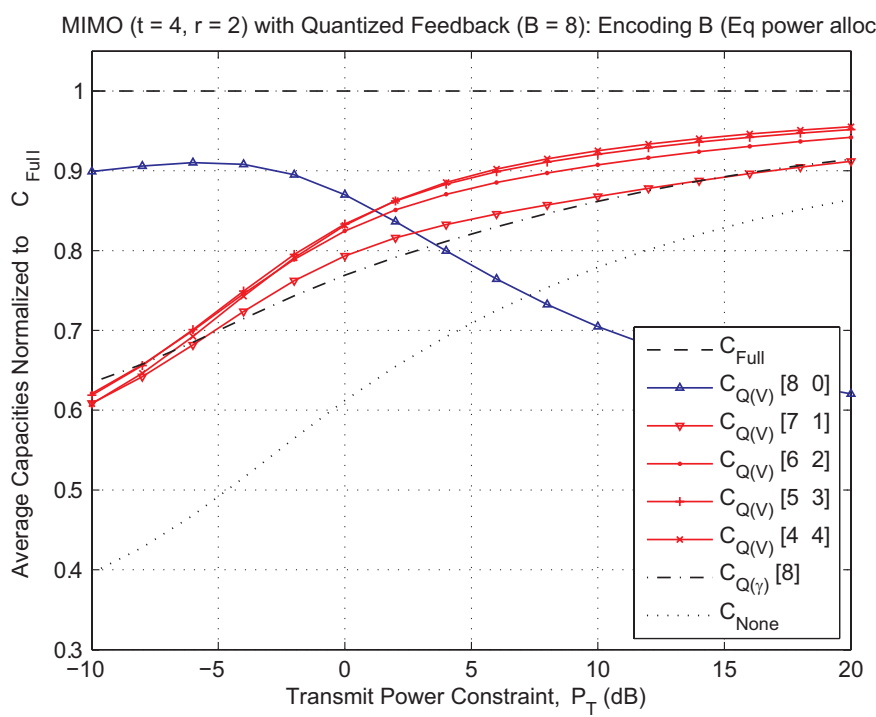

Fig. 4. Normalized average capacities of MIMO channel with quantized feedback: equal power allocation and encoding scheme $\mathrm{B}(t=4, r=2$ and $B=8)$.

design except that code points are matrices and a different design objective is employed.

Fig. 5 shows the performance comparisons with the two matrix-oriented codebook construction methods proposed in [12] and [13], [14]. For fair comparison we consider the sequential VQ employing equal power allocation and encoding scheme $\mathrm{C}$, since in the other two competing methods, only unitary beamforming matrix is quantized and fed back with full search encoding assumed. From the results, it is interesting to see that the sequential VQ provides comparable performance to the MQ-based method with marginal difference. And, the DFT-based method [23] is outperformed by the two codebook constructions (the MQ-based and the sequential VQ method). This is because as also mentioned in [23], the structured codebook designed by the DFT matrix-based algorithm is not necessarily optimal, although the algorithm provides an easy way to obtain the codebook for small number of code points. The figure also shows that with the encoding scheme $\mathrm{C}$, the bit allocation between the extracted unit-norm vectors does not affect the performance. This is in contrast to encoding scheme $\mathrm{B}$ which is sensitive. Although it is hard to tell from the figure, the optimum bit allocation is [4 4 ] $]$ in the most range of SNR and $\left[\begin{array}{ll}5 & 3\end{array}\right]$ at low SNR.

\section{Simple Feedback Method for Slowly TIME-VARYING CHANNELS}

In this section, we introduce another low-complexity quantization scheme based on further parameterization of a vector in terms of a minimal number of independent scalar parameters. In particular, we consider its application to CSI feedback in slowly time-varying MIMO channels.

\section{A. Scalar-Form Parameterization for Spatial Information}

The degrees of freedom in the spatial information matrix is much smaller than the number of real entries in the matrix because of the geometrical structure between the columns of

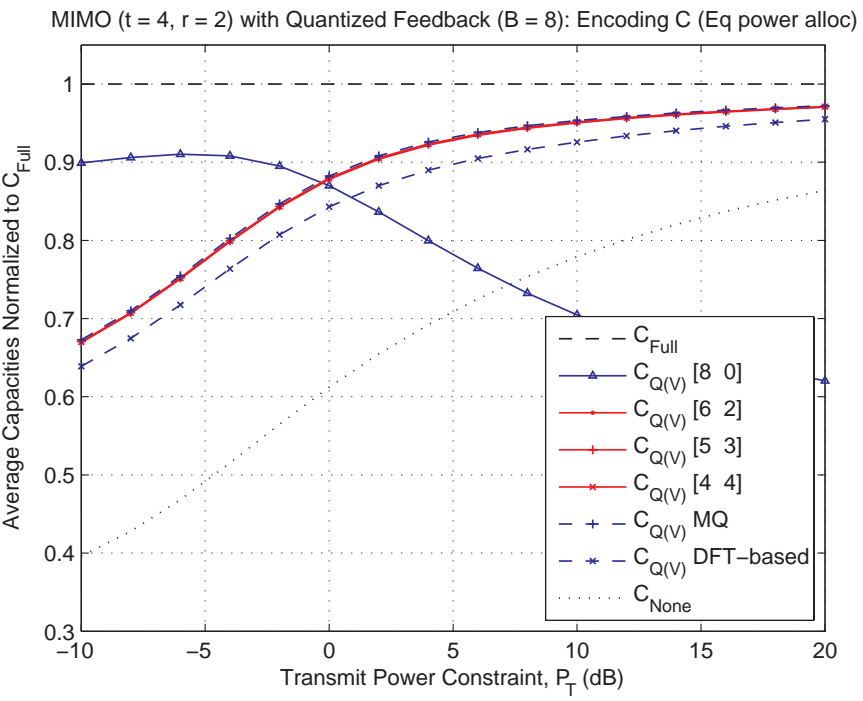

Fig. 5. Normalized average capacities of MIMO channel with quantized feedback: equal power allocation and encoding scheme $\mathrm{C}(t=4, r=2$ and $B=8)$. Codebooks for ' $C_{Q(V)}$ MQ' and ' $C_{Q(V)}$ DFT-based' are designed based on [13] and [12], respectively.

the matrix. For a $t \times n$ orthonormal column matrix, the degrees of freedom (number of free parameters) is given by $N=$ $2 t \cdot n-n-2\left(\begin{array}{c}n \\ 2\end{array}\right)=2 t n-n^{2}$ (real numbers). For example, a $t \times t$ unitary matrix has $2 t^{2}$ real entries, but its degrees of freedom is only $t^{2}$. Furthermore, one phase in each column can be made fixed (e.g., the first row can be chosen to have all nonnegative real numbers), which results in a further reduction in the number of degrees of freedom by $n$, i.e.,

$$
N=(2 t-1) n-n^{2} \text { (real numbers). }
$$

1) Alternate Parameterization: We want to extract a set of essential independent scalar parameters that has a one-toone mapping to the matrix $V$. Conceptually, the vector-form parameterization (in Section III) is further refined: a set of scalar parameters is extracted from each unit-norm vector $q_{i}$. Practically, there are several possible ways. Here we propose a parameterization method using real Givens rotations in which the number of parameters is equal to the minimal number (30). The following theorem summarizes the parameterization method.

Theorem 2: An orthonormal column matrix $V \in \mathbb{C}^{t \times n}(t \geq$ $n$ ) can be decomposed as

$$
V=\left[\prod_{k=1}^{n} D_{k}\left(\phi_{k, k}, \ldots, \phi_{k, t}\right) \prod_{l=1}^{t-k} G_{t-l, t-l+1}\left(\theta_{k, l}\right)\right] \tilde{I}
$$

where $D_{k}\left(\phi_{k, k}, \ldots, \phi_{k, t}\right)=\operatorname{diag}\left(1_{k-1}, e^{j \phi_{k, k}}, \ldots, e^{j \phi_{k, t}}\right)$, $1_{k-1}$ is $(k-1) 1$ 's; and $G_{p-1, p}(\theta)$ is the Givens matrix which operates in the $(p-1, p)$ coordinate plane and is of the form

$$
G_{p-1, p}(\theta)=\left[\begin{array}{cccc}
I_{p-2} & & & \\
& c & -s & \\
& s & c & \\
& & & I_{t-p}
\end{array}\right],
$$

$c=\cos \theta$ and $s=\sin \theta$; and $t \times n$ matrix $\tilde{I}=\left[I_{n}, 0_{n, t-n}\right]^{T}$. 
We clarify the above parameterization procedure with an example. Consider a $4 \times 3$ orthonormal column matrix $V$.

$$
\begin{aligned}
V & =\left[\begin{array}{ccc}
\times & \times & \times \\
\times & \times & \times \\
\times & \times & \times \\
\times & \times & \times
\end{array}\right] \stackrel{D_{1}^{\dagger}}{\longrightarrow}\left[\begin{array}{ccc}
|\times| & \times & \times \\
|\times| & \times & \times \\
|\times| & \times & \times \\
|\times| & \times & \times
\end{array}\right] \stackrel{G_{3,4}^{\dagger}, G_{2,3}^{\dagger}, G_{1,2}^{\dagger}}{\longrightarrow} \\
& {\left[\begin{array}{ccc}
1 & 0 & 0 \\
0 & \times & \times \\
0 & \times & \times \\
0 & \times & \times
\end{array}\right] \stackrel{D_{2}^{\dagger}}{\longrightarrow}\left[\begin{array}{ccc}
1 & 0 & 0 \\
0 & |\times| & \times \\
0 & |\times| & \times \\
0 & |\times| & \times
\end{array}\right] \stackrel{G_{3,4}^{\dagger}, G_{2,3}^{\dagger}}{\longrightarrow} } \\
& {\left[\begin{array}{ccc}
1 & 0 & 0 \\
0 & 1 & 0 \\
0 & 0 & \times \\
0 & 0 & \times
\end{array}\right] \stackrel{D_{3}^{\dagger}}{\longrightarrow}\left[\begin{array}{ccc}
1 & 0 & 0 \\
0 & 1 & 0 \\
0 & 0 & |\times| \\
0 & 0 & |\times|
\end{array}\right] \stackrel{G_{3,4}^{\dagger}}{\longrightarrow} \tilde{I} }
\end{aligned}
$$

In the above, $|\times|$ denotes the magnitude of a particular element. The procedure is similar to the $\mathrm{QR}$ decomposition using Givens matrices [24]. In the first step, we want to make all the entries in the first column under the first component all zeros. To do that, we first extract the phases from the first column by pre-multiplying $V$ by $D_{1}^{\dagger}$ to have a real-valued column, and then apply a series of Givens matrices with appropriate parameters to make all entries under the $(1,1)$ element zeros. Since the Givens rotation preserves the length of vector, the $(1,1)$ element becomes 1 . At the same time, all the entries in the first row except the $(1,1)$ element also become zeros because of the orthogonality between columns (note that unitary matrices preserve orthogonality). We carry out similar procedures on the remaining columns sequentially, and then finally we have a diagonal matrix $\tilde{I}$. Since a Givens matrix is an orthogonal matrix, the matrix $V$ can be factored as

$$
\begin{aligned}
V= & D_{1}\left(\phi_{1,1}, \ldots, \phi_{1,4}\right) G_{3,4}\left(\theta_{1,1}\right) G_{2,3}\left(\theta_{1,2}\right) G_{1,2}\left(\theta_{1,3}\right) \\
& \cdot D_{2}\left(\phi_{2,2}, \phi_{2,3}, \phi_{2,4}\right) G_{3,4}\left(\theta_{2,1}\right) G_{2,3}\left(\theta_{2,2}\right) \\
& \cdot D_{3}\left(\phi_{3,3}, \phi_{3,4}\right) G_{3,4}\left(\theta_{3,1}\right) \tilde{I} .
\end{aligned}
$$

Therefore, once we have a set of parameters, the phases $\left\{\phi_{k, m}\right\}$ and the rotation angles $\left\{\theta_{k, l}\right\}$, the original matrix $V$ can be exactly reconstructed.

The proposed scalar-form parameterization method can be seen as one implementation of vector-form parameterization by setting

$$
\begin{aligned}
& G_{1}=D_{1}\left(\phi_{1,1}, \ldots, \phi_{1, t}\right) G_{t-1, t}\left(\theta_{1,1}\right) \cdots G_{1,2}\left(\theta_{1, t-1}\right), \\
& G_{2}=D_{2}\left(\phi_{2,2}, \ldots, \phi_{2, t}\right) G_{t-1, t}\left(\theta_{2,1}\right) \cdots G_{2,3}\left(\theta_{2, t-2}\right),
\end{aligned}
$$

and so on. It can be easily checked that the unitary matrix $G_{i}$ has the properties mentioned in Section III-A.

Now, we show that the number of essential scalar parameters from those obtained with the parameterization procedure, i.e., $\left\{\phi_{k, m}, \theta_{k, l}: k=1, \ldots, n ; m=k, \ldots, t ; l=1, \ldots, t-\right.$ $k\}$, can be made equal to the degrees of freedom in $V$ with the following theorem.

Theorem 3: In the matrix factorization of Theorem 2, if orthonormal column matrix $V$ has real-valued elements in the first row with alternating signs as $+-+-\ldots$, then the first parameter of $D_{k}$ is zero, i.e., $\phi_{k, k}=0$ for all $k$. Therefore, the

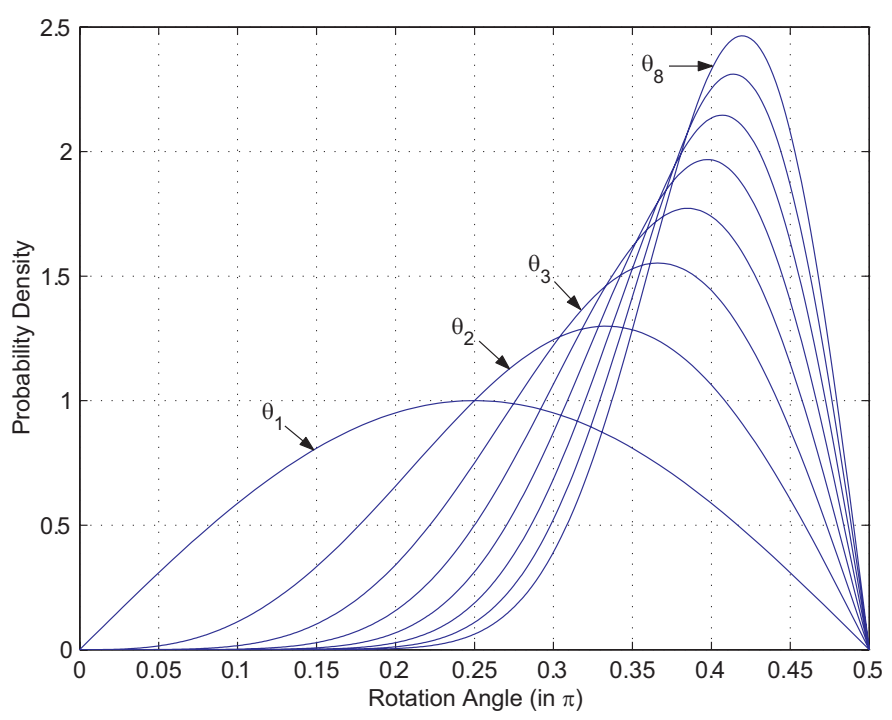

Fig. 6. Probability density functions of rotation angles.

number of parameters is $(2 t-1) n-n^{2}$, which is the degrees of freedom in $V$.

Proof: See [25] for details.

2) Statistical Properties of the Scalar Parameters: In this section, we consider a random MIMO channel with i.i.d. $\tilde{\mathcal{N}}(0,1)$ entries and explore the statistical distribution of the scalar parameters and establish their independence, a property useful for quantization purposes.

For simplicity, we start with MISO channels and later extend to MIMO channels. Consider a random MISO channel $h$ with i.i.d. entries and apply the parameterization procedure to $v=h /\|h\|$. Let us denote the scalar parameters by $\phi_{1}, \ldots, \phi_{t}$ (phases) and $\theta_{1}, \ldots, \theta_{t-1}$ (rotation angles). Obviously, the phases $\phi_{i}$ are all independent and each uniformly distributed over $(-\pi, \pi]$. For the distribution of the rotation angles, we can derive the following result.

Lemma 3: The rotation angles $\theta_{1}, \ldots, \theta_{t-1}$ are statistically independent and $\theta_{l}$ has the density function

$p\left(\theta_{l}\right)=2 l \sin ^{2 l-1} \theta_{l} \cos \theta_{l}, 0 \leq \theta_{l}<\frac{\pi}{2}$, for $l=1, \ldots, t-1$.

Proof: An interesting and useful fact is that, when the parameterization procedure is applied to the unnormalized vector $h$, the Givens rotation angles are same as those when the procedure is applied to the normalized vector $v$. We can find the probability densities for the rotation angles $\left\{\theta_{i}\right\}$ using techniques for calculating the distribution of transformed random vector [22, ch. 2], especially using the Jacobian of a transformation given in Theorem 2.1.3 of [22]. A detailed proof of the lemma is provided in [25].

Fig. 6 shows the density functions of the rotation angles, where we can see that as the index $l$ increases the angle is more concentrated.

Now we extend the statistical results for MISO channels to MIMO channels.

Theorem 4: When the channel matrix $H$ has i.i.d. $\tilde{\mathcal{N}}(0,1)$ entries, then all the parameters from Theorem 2 are statistically independent. Moreover, the phase $\phi_{k, j}$ is uniformly distributed over $(-\pi, \pi]$ for all $k$ and $j$, and the rotation angle 
$\theta_{k, l}$ has probability density

$$
\begin{aligned}
p\left(\theta_{k, l}\right)= & 2 l \sin ^{2 l-1} \theta_{k, l} \cos \theta_{k, l}, 0 \leq \theta_{k, l}<\frac{\pi}{2}, \\
& \quad \text { for } 1 \leq k \leq n, 1 \leq l \leq t-k .
\end{aligned}
$$

Proof: For a random MIMO channel $H$ with i.i.d. $\tilde{\mathcal{N}}(0,1)$ entries, it has been shown that the right singular matrix $V_{H} \sim \mathcal{U}\left(\mathcal{V}_{m, t}\right)$, and $V \sim \mathcal{U}\left(\mathcal{V}_{n, t}\right)$. In order to obtain the density functions of the scalar parameters, we first recall from Section III-A that $V$ can transformed into a sequence of independent vectors $q_{i} \in \mathcal{S}_{t-i+1}$. Each of the $q_{i}$ 's can be further transformed into independent phases and rotation angles as indicated in Lemma 3. Collecting all the parameters and noting the independence of the $q_{i}$ 's and, hence, of the corresponding scalar parameters (phases and rotation angles), by Lemma 3, we have the above stated result.

3) Parameterization for Power Allocation: The parameterization for power allocation information $\gamma=\left[\gamma_{1}, \ldots, \gamma_{t}\right]$, $\sum_{i=1}^{t} \gamma_{i}=1$ is treated in a rather simple manner. We can see that $\gamma$ has $t-1$ of degrees of freedom. And, the parameters can be simply the first $t-1$ elements, $\left[\gamma_{1}, \ldots, \gamma_{t-1}\right]$. Then, from the constraint, the last one is determined as $\gamma_{t}=1-\sum_{i=1}^{t-1} \gamma_{i}$.

\section{B. Simple Differential Feedback Scheme}

The feedback strategy depicted in Fig. 2 that is based on quantization in the transformed parameter domain is also considered in this section. The proposed methodology is general and can be applied to any multiple antenna scenario: MISO systems (when $n=1$ ) and MIMO systems with partial feedback (when $1<n<m$ ) as well as MIMO systems with full feedback $(n=m)$. One can apply a VQ method to quantize the parameters. But, complexity issues and tracking requirement motivate us to consider employing scalar quantization. Moreover, the independence of the parameters (Theorem 4) indicates that the overall loss is minimal. More specifically, adaptive delta modulation (ADM) [26, ch. 8] is used to quantize each parameter. The following observation about the temporal behavior of the extracted parameters in slowly time-varying channels motivates us to consider ADM. In slowly time-varying channels, the parameters are also slowly and continuously changing most of the time. The encoder of ADM quantizes the difference between the newly incoming sample and the previous quantized sample. For a parameter $\theta$,

$$
\hat{\theta}[k]=\hat{\theta}[k-1] \pm \Delta[k] .
$$

The step-size $\Delta[k]$ of the one-bit quantizer is adaptively changing to better track the dynamics of the signal. The ADM with one-bit memory [26] is an example. The step-size is increased if two successive encoded bits are the same, and decreased otherwise, that is,

$$
\Delta[k]= \begin{cases}M_{1} \Delta[k-1], & \text { if } c[k]=c[k-1] \\ M_{2} \Delta[k-1], & \text { if } c[k] \neq c[k-1]\end{cases}
$$

where $\Delta[k]$ and $c[k]$ is the step-size and the encoded bit for the $k$ th sample; and $M_{1}>1$ and $0<M_{2}<1$, usually $M_{2}=1 / M_{1}$. Compared to $\mathrm{VQ}$, ADM has considerably lower complexity. Another important advantage is that ADM has inherently a channel tracking feature for slowly time-varying channels. Some simulation results are shown in Figs. 7 and 8 for temporarily correlated MIMO channel. As seen from the figures, in slowly time-varying channels, with ADM, we can track the extracted parameters well. A closer examination of Fig. 8 reveals that there are several jumps in phases. The jumps occur during the time when an entry of the spatial information matrix $V$ has small magnitude and is varying around the origin resulting in a fast changing phase.

\section{Simulation Results}

We describe a more comprehensive feedback system model for evaluating feedback in time-varying MIMO channels that accounts for the discrepancy between the real channel and the CSI at the transmitter.

1) Feedback System Model: Fig. 9 depicts the block-fading model and the frame structure of the feedback system model. We assume that the channel matrix is not changing during a time block of length $T_{C}$, which will be called channel block length. In the next channel block, we have a different channel realization following the temporal correlation characteristics of the fading channel. The channel matrix for $k$ th channel block is denoted by $H[k]$, and $V[k]$ and $\gamma[k]$ are the corresponding CSI. The quantized version of the CSI ( $\hat{V}[k]$ and $\hat{\gamma}[k]$ in the figure) is provided to the transmitter at a feedback rate of $R_{F}$ times per second via a feedback channel. The time frame between two consequent channel updates is called feedback frame (with length $T_{F}=1 / R_{F}$ ). For simplicity, we assume there are $M$ (an integer) channel blocks in a feedback frame, i.e., $T_{F}=M T_{C}$. This means that during a feedback frame, the channel changes $M$ times, which models the dynamics of the fading channel. In addition, in order to model composite delay, e.g., due to processing and propagation, we also introduce an integer parameter $D$ : at the starting point of each feedback frame, the CSI corresponding to the $D$ previous channel block is available at the transmitter. Fig. 9 is an example when $D=$ 1. The CSIT is used in transmission during the frame before the next update arrives.

2) Simulation Results: We have performed simulations to investigate the performance of the proposed feedback method, especially in slowly time-varying MIMO channels. The components of the channel matrix are i.i.d. discrete-time random processes and each process models Rayleigh fading channel gain. The channel was simulated using the conventional Jakes model with a Doppler frequency $f_{D}=7.4 \mathrm{~Hz}$, which corresponds to a mobility of $4 \mathrm{~km} / \mathrm{h}$ at carrier frequency of $2 \mathrm{GHz}$. As for the frame structure of the feedback system model, we considered the case of $M=4$ and $D=1$.

Fig. 10 shows the cumulative distribution of mutual information with different CSIT assumptions and various transmit power, $P_{T}=-10,0,10,20 \mathrm{~dB}$, with the feedback rate $R_{F}=500$ times per second $(t=4$ and $r=2)$. $C_{\text {Full }}$ and $C_{\text {None }}$ are the capacity for the full and no CSIT, respectively. Note that the CSIT for $C_{\text {Full }}$ is perfect, that is, it involves neither quantization error nor channel tracking error. The performances of the proposed feedback method are shown as $C_{\hat{V}, \hat{\gamma}}$ and $C_{\hat{V}}$, which are for the cases of feeding back the quantized $(V, \gamma)$ and the quantized $V$ only, respectively, using the given fixed number of feedback bits. These include the 
Rotation Angles: $M_{1}=12$
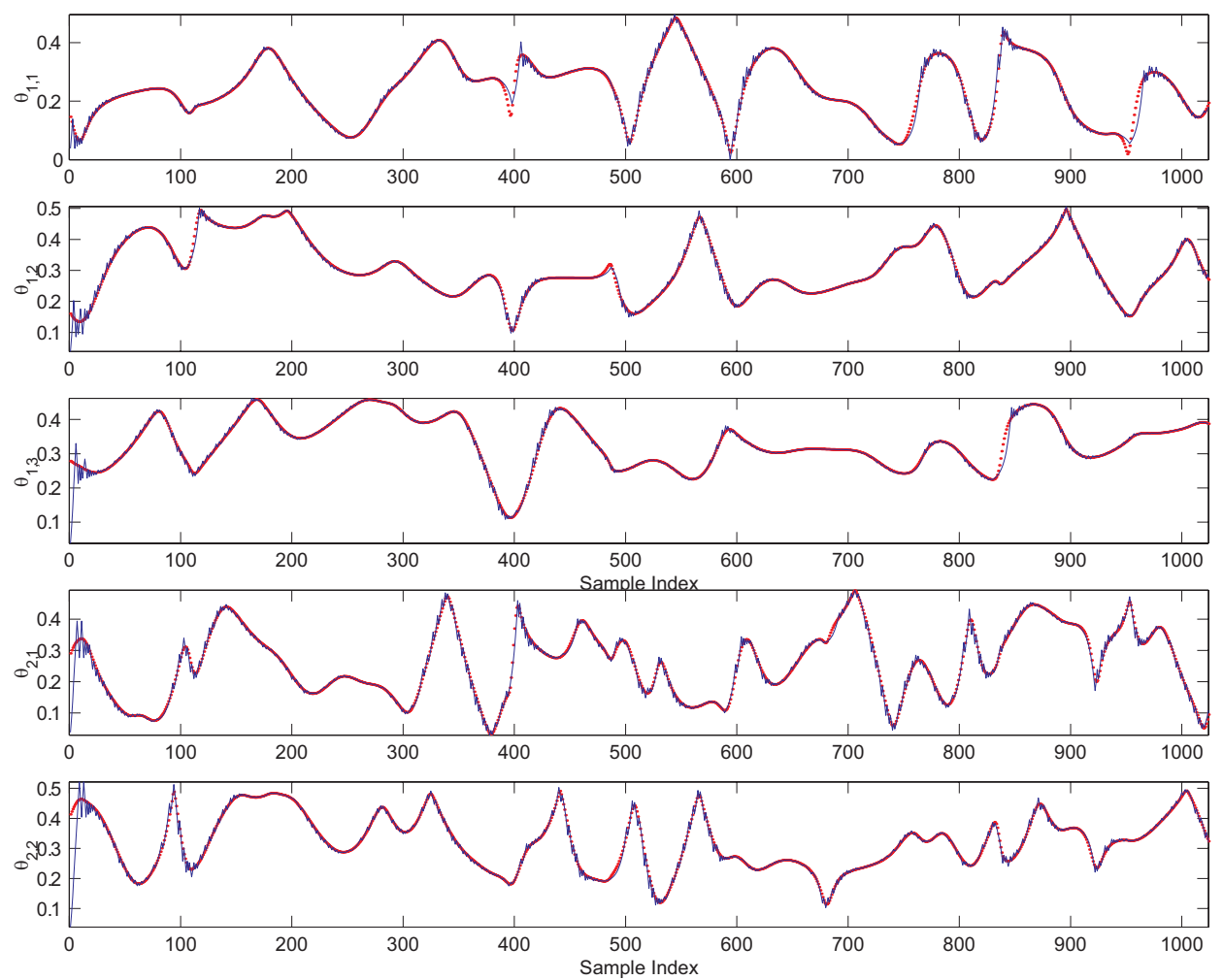

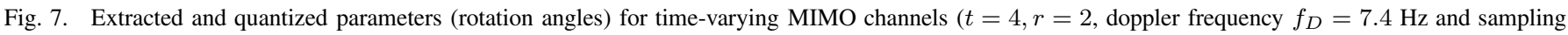
frequency is 1,000 times per second). The dotted (solid) lines are for extracted (quantized) parameters in $\pi$.
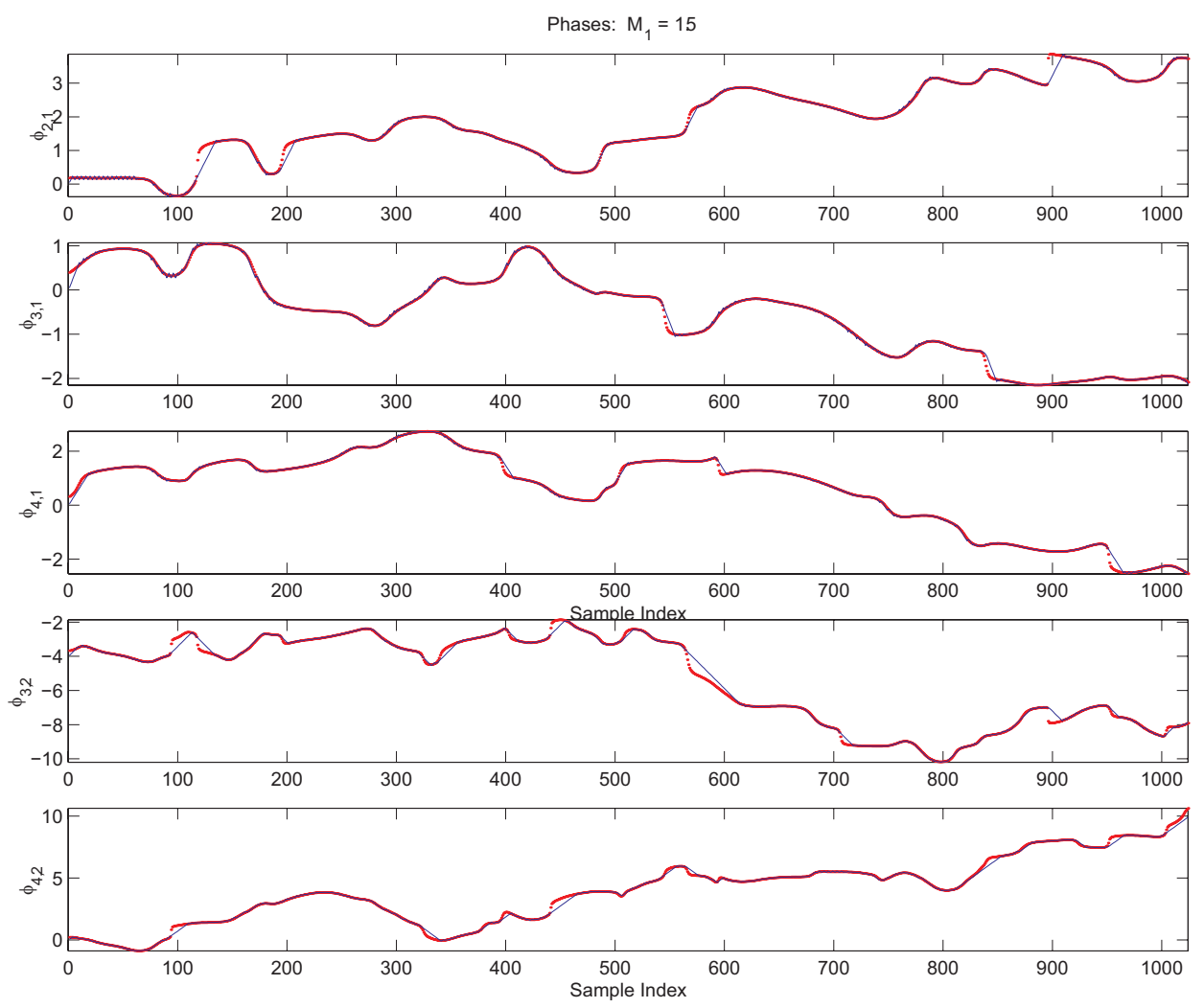

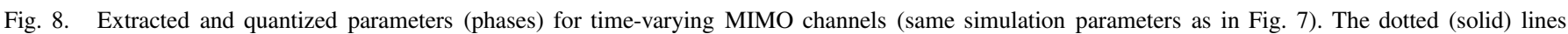
are for extracted (quantized) parameters in $\pi$. 


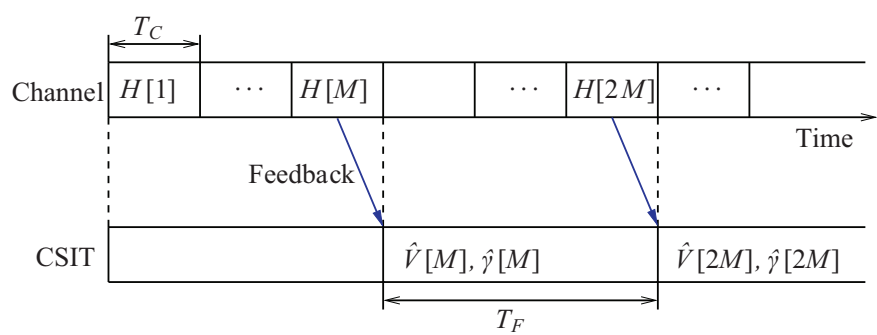

Fig. 9. Feedback system model (when $D=1$ ).

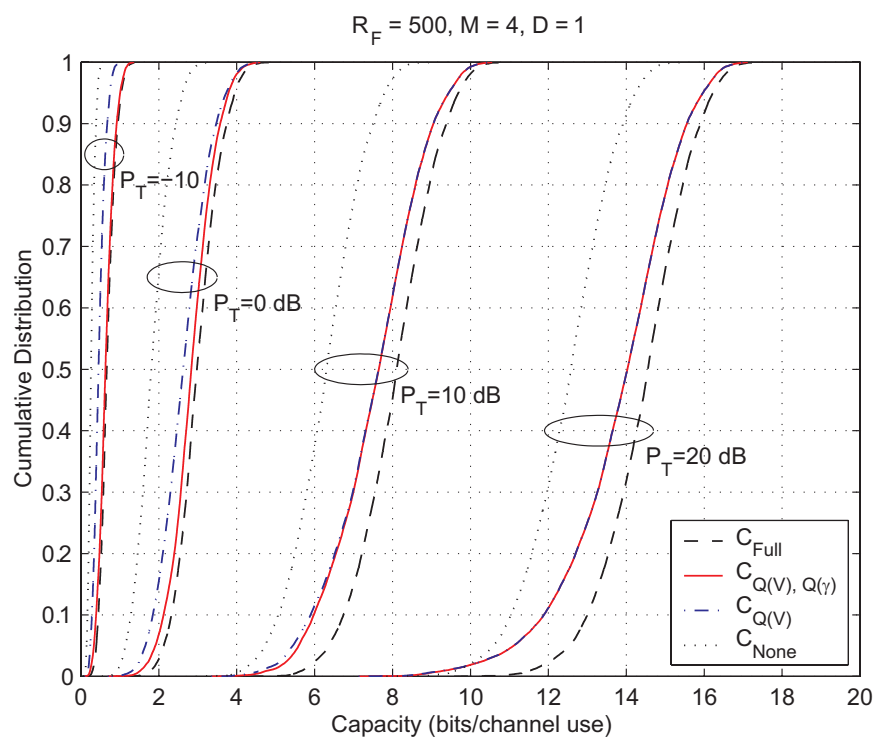

Fig. 10. Cumulative distribution of capacities when $R_{F}=500$ times per $\sec (t=4$ and $r=2) . Q(V)=\hat{V}$ and $Q(\gamma)=\hat{\gamma}$.

effect of quantization error and delay; therefore, they reflect more practical and realistic situations for feedback systems. From the results, we can see that, in low transmit power range, the two have some gap; but, in high transmit power range, the two have little difference. This means that power allocation information is important in low transmit power range which is supported by the water-filling argument. That is, when transmit power is low, the optimum transmission scheme uses only a few spatial channels that have high channel gains. Note that the feedback rate is corresponding to $5.5 \mathrm{kbps}$ (for $C_{\hat{V}, \hat{\gamma}}$ ) and $5 \mathrm{kbps}$ (for $C_{\hat{V}}$ ) of feedback bit-rate, since we have 10 parameters for $V$ and one for $\gamma$, and ADM encodes each parameter using one bit at each feedback instant.

Fig. 11 shows the results when the feedback rate is increased to $R_{F}=1,000$ times per second, which corresponds to $11 / 10$ kbps. We can see that the performances improves and becomes much closer to $C_{\text {Full }}$. This is as expected since by increasing the feedback rate, the quantization error is reduced, and hence in ADM encoding the variation between the adjacent samples is reduced. Also the channel tracking error due to delay is reduced by increasing the feedback rate.

\section{SUMmARY}

We introduced a general framework for quantization and feedback of MIMO channel information, which involves parameterization and quantization in the parameter domain. The

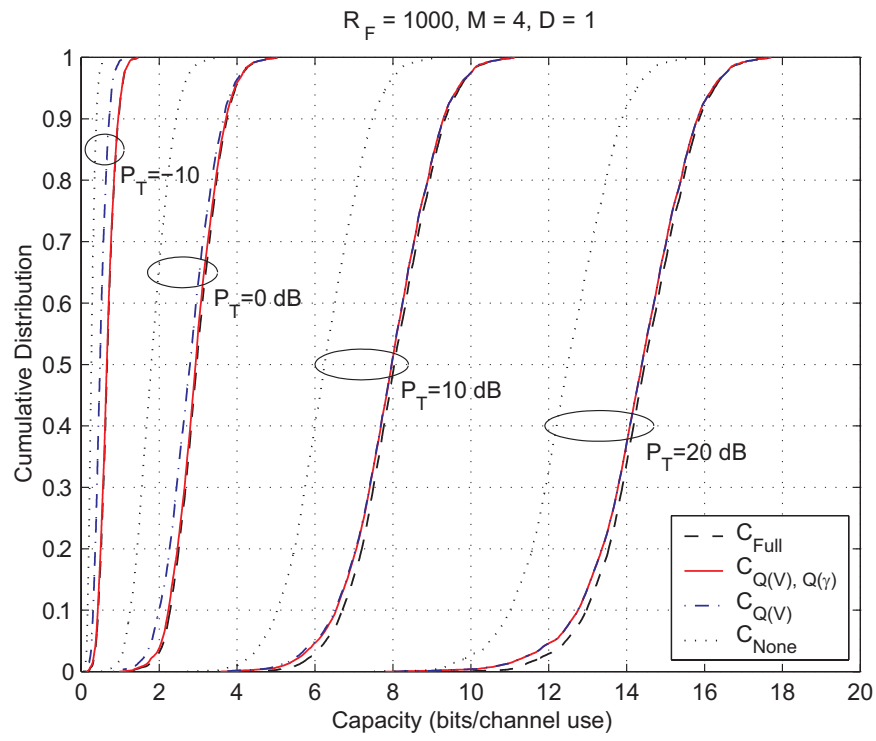

Fig. 11. Cumulative distribution of capacities when $R_{F}=1,000$ times per $\sec (t=4$ and $r=2)$.

proposed parameterization methods exploit the geometrical structure of orthonormality while quantizing the spatial information matrix. The parameterization method can be easily implemented using Givens rotations. In the first feedback scheme for instantaneous channel feedback, the parameter vectors (unit-norm) are quantized using a vector quantization technique. This sequential VQ method provides a unique approach to construct the beamforming-matrix codebook, and the different encoding schemes provide a mechanism to tradeoff complexity for performance. The statistical properties of the extracted parameters were found such as the independence between parameters and the exact distribution for scalar parameters. In slowly time-varying channels, the scalar parameters are also found to be slowly and continuously changing over time. This motivated a simple form of differential encoding scheme, adaptive delta modulation, in quantizing the parameters. As a natural extension, the differential scalar quantization method can be generalized to differential vector quantization.

\section{ACKNOWLEDGMENT}

The authors would like to thank the anonymous reviewers and Associated Editor Dr. Vincent Lau for helpful comments, and Dr. David Love for providing the codebooks based on [12].

\section{REFERENCES}

[1] A. Goldsmith, S. A. Jafar, A. Jindal, and S. Vishwanath, "Capacity limits of MIMO channels," IEEE J. Select. Areas Commun., vol. 21, no. 5, pp. 684-702, June 2003.

[2] A. Narula, M. J. Lopez, M. D. Trott, and G. W. Wornell, "Efficient use of side information in multiple-antenna data transmission over fading channels," IEEE J. Select. Areas Commun., vol. 16, pp. 1423-1436, Oct. 1998.

[3] K. K. Mukkavilli, A. Sabharwal, E. Erkip, and B. Aazhang, "On beamforming with finite rate feedback in multiple antenna systems," IEEE Trans. Inform. Theory, vol. 49, no. 10, pp. 2562-2579, Oct. 2003.

[4] J. C. Roh and B. D. Rao, "Performance analysis of multiple antenna systems with VQ-based feedback," in Proc. Asilomar Conf., Nov. 2004, pp. 1978-1982. 
[5] — , "Transmit beamforming in multiple-antenna systems with finite rate feedback: A VQ-based approach," IEEE Trans. Inform. Theory, vol. 52, no. 3, pp. 1101-1112, Mar. 2006.

[6] P. Xia and G. B. Giannakis, "Design and analysis of transmitbeamforming based on limited-rate feedback," in Proc. IEEE Vehicular Technology Conf., Sep. 2004, pp. 1653-1657.

[7] P. Xia, S. Zhou, and G. B. Giannakis, "Achieving the Welch bound with difference sets," in Proc. IEEE ICASSP, Mar. 2005, vol. 3, pp. 10571060.

[8] D. J. Love, R. Heath, Jr., and T. Strohmer, "Grassmannian beamforming for multiple-input multiple-output wireless systems," IEEE Trans. Inform. Theory, vol. 49, no. 10, pp. 2735-2747, Oct. 2003.

[9] D. J. Love and R. Heath, Jr., "Limited feedback precoding for spatial multiplexing systems," in Proc. IEEE GLOBECOM, Dec. 2003, vol. 4, pp. 1857-1861.

[10] W. Santipach and M. L. Honig, "Asymptotic performance of MIMO wireless channels with limited feedback," in Proc. IEEE MILCOM, Oct. 2003, pp. 141-146.

[11] V. K. N. Lau, Y. Liu, and T. A. Chen, "On the design of MIMO blockfading channels with feedback-link capacity constraint," IEEE Trans. Commun., vol. 52, no. 1, pp. 62-70, Jan. 2004.

[12] D. J. Love and R. Heath, Jr., "Limited feedback unitary precoding for orthogonal space-time block codes," IEEE Trans. Signal Processing, vol. 53, no. 1, pp. 64-73, Jan. 2005.

[13] J. C. Roh and B. D. Rao, "Design and analysis of MIMO spatial multiplexing systems with quantized feedback," IEEE Trans. Signal Processing, vol. 54, no. 8, pp. 2874-2886, Aug. 2006.

[14] - "MIMO spatial multiplexing systems with limited feedback," in Proc. IEEE Int. Conf. Commun., May 2005, pp. 777-782.

[15] W. Dai, Y. Liu, B. Rider, and V. K. N. Lau, "On the information rate of MIMO systems with finite rate channel state feedback and power on/off strategy," in Proc. ISIT, Sep. 2005, pp. 1549-1553.

[16] A. Gersho and R. M. Gray, Vector Quantization and Signal Compression. Norwell, MA: Kluwer Academic, 1992.

[17] R. M. Gray and D. L. Neuhoff, "Quantization," IEEE Trans. Inform. Theory, vol. 44, no. 6, pp. 2325-2383, Oct. 1998.

[18] C. Murthy, J. C. Roh, and B. D. Rao, "Optimality of extended maximum ratio transmission," in 6th Baiona Workshop on Signal Processing in Commun., Sep. 2003, pp. 47-50.

[19] J. C. Roh and B. D. Rao, "Multiple antenna channels with partial channel state information at the transmitter," IEEE Trans. Wireless Commun., vol. 3, no. 2, pp. 677-688, Mar. 2004.

[20] A. Edelman, "Eigenvalues and Condition Numbers of Random Matrices." Ph.D. diss., Massachusetts Institute of Technology, 1989.

[21] T. L. Marzetta and B. M. Hochwald, "Capacity of a mobile multiple- antenna communication link in Rayleigh flat fading," IEEE Trans. Inform. Theory, vol. 45, pp. 139-157, Mar. 1999.

[22] R. J. Muirhead, Aspects of Multivariate Statsitical Theory. New York: John Wiley \& Sons, 1982.

[23] B. M. Hochwald, T. Marzetta, T. Richardson, W. Sweldens, and R. Urbanke, "Systematic design of unitary space-time constellations," IEEE Trans. Inform. Theory, vol. 46, pp. 1962-1973, Sep. 2000.

[24] G. H. Golub and C. F. Van Loan, Matrix Computations, 3rd ed. Baltimore, MD: Johns Hopkins University Press, 1996.

[25] J. C. Roh, "Multiple-Antenna Communication Systems with Finite Rate Feedback." Ph.D. diss., University of California at San Diego, Apr. 2005.

[26] N. S. Jayant and P. Noll, Digital Coding for Waveforms: Principles and Applications to Speech and Video. Upper Saddle River, NJ: PrenticeHall, 1984.

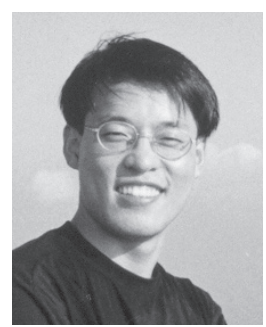

June Chul Roh (S'03-M'06) received the B.S. and M.S. degrees in electronics engineering from the University of Seoul, Seoul, Korea, in 1993 and 1995, respectively, and the Ph.D. degree in electrical engineering from the University of California, San Diego, La Jolla, CA, in 2005.

From 1995 to 2000 , he was a Member of Technical Staff at Korea Telecom R\&D Group, Seoul, Korea, where he worked on third-generation cellular systems and was involved in developing a wideband CDMA system. He is currently with Texas Instruments, Inc., Sunnyvale, CA. His research interests include communication theory, information theory, statistical signal processing, multiple-antenna systems, CDMA and OFDM systems.

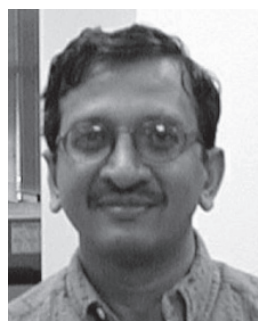

Bhaskar D. Rao (S'80-M'83-SM'91-F'00) received the B.Tech. degree in electronics and electrical communication engineering from the Indian Institute of Technology, Kharagpur, India, in 1979 and the M.S. and Ph.D. degrees from the University of Southern California, Los Angeles, in 1981 and 1983, respectively.

Since 1983, he has been with the University of California, San Diego, La Jolla, where he is currently a Professor with the Electrical and Computer Engineering Department. His interests are in the areas of digital signal processing, estimation theory, and optimization theory, with applications to digital communications, speech signal processing, and human-computer interactions.

Dr. Rao has been a member of the Statistical Signal and Array Processing Technical Committee of the IEEE Signal Processing Society. He is currently a member of the Signal Processing Theory and Methods Technical Committee. 